Article

\title{
Modification of a Phenolic Resin with Epoxy- and Methacrylate-Functionalized Silica Sols to Improve the Ablation Resistance of Their Glass Fiber-Reinforced Composites
}

Yu Hu ${ }^{1, *}$, Wenlong Geng ${ }^{2}$, Hong You ${ }^{2}$, You Wang ${ }^{1, *}$ and Douglas A. Loy ${ }^{3}$

1 School of Materials Science and Engineering, Harbin Institute of Technology, Harbin 150001, China

2 State Key Laboratory of Urban Water Resource and Environment, Harbin Institute of Technology, Harbin 150001, China; E-Mails: hulijiang2006@126.com (W.G.); youhong@hit.edu.cn (H.Y.)

3 Department of Materials Science and Engineering, the University of Arizona, Tucson, AZ 85721, USA; E-Mail: daloy@mse.arizona.edu

* Authors to whom correspondence should be addressed; E-Mails: yuhu0104@hit.edu.cn (Y.H.); y-wang@hit.edu.cn (Y.W.); Tel.: +86-451-8641-2679 (Y.H.); Fax: +86-451-8622-1048 (Y.H.).

Received: 16 December 2013; in revised form: 26 December 2013 / Accepted: 30 December 2013 / Published: 6 January 2014

\begin{abstract}
Functionalized silica sols were obtained by the hydrolytic condensation of ( $\gamma$-methacryloxypropyl)trimethoxysilane (MPMS), ( $\gamma$-glycidyloxypropyl)trimethoxysilane (GPMS) and tetraethoxysilane (TEOS). Three different sols were obtained: MPS (derived from MPMS and TEOS), GPS-MPS (derived from GPMS, MPMS and TEOS), and GPSD (derived from GPMS, TEOS and diglycidyl ether of bisphenol A, DGEBA). These silica sols were mixed with a phenolic resin (PR). Ethylenediamine was used as a hardener for epoxy-functionalized sols and benzoyl peroxide was used as an initiator of the free-radical polymerization of methacrylate-functionalized silica sols. Glass fiber-reinforced composites were obtained from the neat PR and MPS-PR, GPS-MPS-PR and GPSD-PR. The resulting composites were evaluated as ablation resistant materials in an acetylene-oxygen flame. A large increase in the ablation resistance was observed when the PR was modified by the functionalized silica sols. The ablation resistance of the composites decreased as follows:
\end{abstract} GPSD-PR $>$ MPS-PR $>$ GPS-MPS-PR $>$ PR.

Keywords: ablation; glass fiber-reinforced composite; phenolic resin (PR); silica sol; silsesquioxanes; MPMS; GPMS; TEOS; DGEBA 


\section{Introduction}

The ability of composites based on phenolic resins and glass or carbon fibers to withstand extremely high heat flux for short periods has made their use for re-entry nose cones and rocket nozzles possible [1]. The combined effects of heat and stress, termed ablation, involve raising only a thin surface layer of material to a high temperature, keeping the bulk of the material unexposed to the damaging temperatures [2-5]. Reinforced phenolic composites have been extensively used as ablation materials on intercontinental missile warheads and space shuttles [6]. However, there is still need to improve their ablation resistance, a fact that can be accomplished by a convenient design of the chemical structure of the matrix [7], or the cure cycle [8].

Two types of phenolic resins are used as matrices of ablation-resistant composites. Novolacs are phenolic resins made by the condensation of phenol with a defect of formaldehyde. They are obtained as powders and are usually called two-step phenolic resins because they need to be cross-linked with a suitable hardener in a second step. Hexamethylenetetramine (also a powder) is the most typical hardener used in industry. The other types of phenolic resins are resoles which are obtained by condensation of phenol with an excess of formaldehyde. They are called one-step phenolic resins because they continuously undergo polymerization leading to a cross-linked product without the need of adding an extra hardener. They are obtained as liquid solutions in water and must be stored at low temperatures to avoid gelation.

Different modifiers have been used to increase the ablation resistance of phenolic resins. For example, boron-modified phenolic resins exhibit outstanding ablation performance [9]. However, many other types of modifiers such as silica precursors can also be used for this purpose. It has been reported that modification of thermosetting polymers with polyhedral oligomeric silsesquioxanes (POSS), or silsesquioxanes and functionalized silica sols, increases the mechanical and thermal properties of the cross-linked polymer and the residual mass after degradation at high temperatures [10-15]. Silsesquioxanes (or polysilsesquioxanes) are the condensation products of organotrialkoxysilanes [10,16-18], while functionalized silica sols are obtained by the co-condensation of organotrialkoxysilanes with a silica precursor such as tetraethoxysilane (TEOS) [12,13]. Functionalization of either the silsesquioxane or the silica sol is generated by the organic group present in the chemical structure of the organotrialkoxysilane. This organic group may be reactive or non-reactive with the rest of the components of the formulation. Alkyl groups (e.g., methyl, propyl, isobutyl, etc.) are typical non-reactive species as a variety of reactive organic groups (e.g., epoxy, vinyl, methacrylate, etc.) may be selected depending on the chemical reactions necessary to crosslink the functionalized silica sol with the rest of the components of the formulation.

In a recent publication it was shown that addition of silsesquioxane bearing non-reactive methyl groups produced an enhancement of the ablation resistance of a phenolic resin/carbon fiber composite [19]. Following these results, the aim of this study was to investigate the effect of introducing a reactive functional group in a functionalized silica sol used as a modifier of a phenolic resin/glass fiber composite. The phenolic resin was a commercial novolac provided as a powder containing hexamethylenetetramine as a hardener. Two strategies were used to produce the chemical bonding of the functionalized silica sol with the phenolic novolac. The first strategy consisted in using an organotrialkoxysilane with the organic group containing an epoxy ring. Specifically, 
( $\gamma$-glycidyloxypropyl)trimethoxysilane (GPMS) was co-condensed with tetraethoxysilane (TEOS) in the presence of diglycidyl ether of bisphenol A (DGEBA), to generate a silica sol functionalized with epoxy groups (GPSD, Figure 1A). Apart from GPSD, a diamine (ethylenediamine, EDA) was added to the formulation to partially crosslink the epoxy-functionalized silica sol and DGEBA, by a typical epoxy-amine reaction [20]. This reaction generates a tertiary amine which in turn acts as a catalyst for the reaction of the epoxy excess with the $\mathrm{OH}$ groups of the phenolic resin [21]. This enabled to produce the desired covalent bonds among the functionalized silica sol, DGEBA and phenolic resin.

Figure 1. Preparation of GPSD [derived from ( $\gamma$-glycidyloxypropyl)trimethoxysilane (GPMS), tetraethoxysilane (TEOS) and diglycidyl ether of bisphenol A (DGEBA)] (A); MPS [derived from ( $\gamma$-methacryloxypropyl)trimethoxysilane (MPMS) and TEOS] (B) and GPS-MPS (derived from MPMS, GPMS and TEOS) (C) sols.



(B)



(C)<smiles>[2H][SiH2]O[GaH2]</smiles>

TEOS

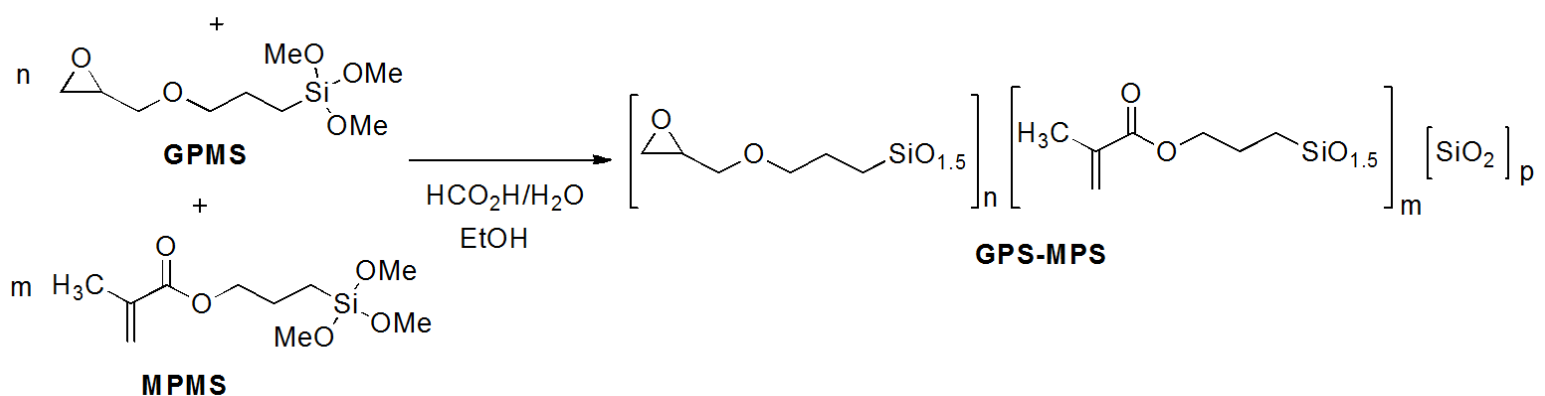

The second strategy was to synthesize a functionalized silica sol by the co-condensation of ( $\gamma$-methacryloxypropyl)trimethoxysilane (MPMS) with TEOS (MPS, Figure 1B). Benzoyl peroxide (BPO) was added to the final formulation to produce the cross-linking of MPS by a free-radical polymerization reaction. The covalent bonding of the functionalized silica sol with the phenolic resin was assumed to be achieved by typical chain-transfer reactions produced by the abstraction of hydrogen radicals from the phenolic novolac.

For comparison purposes, a functionalized silica sol was obtained by co-condensation of MPMS, GPMS and TEOS (GPS-MPS, Figure 1C). In this case both EDA and BPO were used to produce the crosslinking reactions. Glass fiber-reinforced composites were obtained from the neat phenolic resin 
(PR) and MPS-PR, GPS-MPS-PR and GPSD-PR and the corresponding hardeners (EDA and/or BPO). The ablation resistance of the different composites was analyzed and compared with the behavior of the composite derived from the neat phenolic resin.

\section{Experimental Section}

\subsection{Materials}

Commercial GPMS (Dow Corning Z-6040, Beijing, China), MPMS (Dow Corning Z-6030, Beijing, China) and TEOS (96.0\%-97.0\%) were used as precursors of the functionalized silica sols. Diglycidyl ether of biphenol A (DGEBA, 95.0\%), ethanol (99.7\%), tetrahydrofuran (THF, 99.7\%) and formic acid ( $88 \mathrm{wt} \%$ ) were purchased from the Tianjin Chemicals, Tianjin, China. EDA and BPO were analytical grade reagents obtained from the Tianjin Chemicals. Glass fibers (vitreous silica fabrics, VSF) and the phenolic resin (PR, based on a novolac with $M_{\mathrm{w}}: 500-900 \mathrm{~g} / \mathrm{mol}$ together with hexamethylenetetramine as a hardener), were also obtained from the Tianjin Chemicals.

\subsection{Preparation of Composites}

The functionalized silica sols (GPSD, MPS and GPS-MPS) were prepared following procedures described in the literature $[12,13]$. Briefly, formic acid (50 mol \% relative to total $\mathrm{Si}$ ) and water (100 mol \% relative to total $\mathrm{Si}$ ) produced the hydrolytic condensation of TEOS (15 wt \%) with GPMS (15 wt \%), in the presence of DGEBA, to afford GPSD, or with MPMS (15 wt \%) to afford MPS, or with GPMS (7.5 wt \%) and MPMS (7.5 wt \%) to afford GPS-MPS. The addition of DGEBA to GPMS reduces the hydrolysis or alcoholysis of epoxy rings during the hydrolytic condensation [12]. The hydrolytic condensation led to soluble products, despite the use of significant amounts of TEOS [22], due to the network blocking characteristics of the GPMS and MPMS monomers (they are trifunctional monomers while TEOS is a tetrafunctional monomer), and the low water to silicon ratio $(1: 1)$ used [23]. The large fraction of intramolecular cycles produced in these polymerizations also favours the generation of soluble products $[12,16-18]$.

The functionalized silica sols (20 wt \%) were mixed with the PR powder ( $80 \mathrm{wt} \%)$, stirred for $8 \mathrm{~h}$ at $65{ }^{\circ} \mathrm{C}$ and diluted with 10 volumes of ethanol. Glass fabrics $(96 \mathrm{~mm} \times 96 \mathrm{~mm})$ were impregnated with this solution. Ethanol was removed by drying at room temperature for $1 \mathrm{~h}$ and at $60{ }^{\circ} \mathrm{C}$ for $10 \mathrm{~min}$. Twenty of these pre-impregnated glass fabrics were stacked together, coating every layer with the solution of the PR with the functionalized silica sol and the appropriate hardener. EDA was incorporated to formulations containing epoxy groups (molar ratio $\mathrm{Si} / \mathrm{EDA}=4 / 1$ ), and BPO to formulations containing methacrylate groups (in a weight ratio 1:100 with respect to MPMS). The cure was performed in the oven of a universal testing machine (United Testing Systems, Model STM $50 \mathrm{KN}$, Huntington Beach, CA, USA), using the following thermal cycle: $30 \mathrm{~min}$ at $100{ }^{\circ} \mathrm{C}, 60 \mathrm{~min}$ at $130{ }^{\circ} \mathrm{C}$ at a pressure of $5 \mathrm{MPa}, 120 \mathrm{~min}$ at $180{ }^{\circ} \mathrm{C}$ and $10 \mathrm{MPa}, 30 \mathrm{~min}$ at $100^{\circ} \mathrm{C}$ keeping the pressure at $10 \mathrm{MPa}$, followed by the cooling to room temperature. 


\subsection{Characterization and Measurements}

For the ablation tests, the samples were placed at $20 \mathrm{~cm}$ from the flame of the ablation gun originated by the combustion of acetylene with oxygen, both at a pressure of $0.5 \mathrm{MPa}$. Samples were exposed for $30 \mathrm{~s}$ and both linear and mass ablation values were determined. The evolution of temperature was recorded at twenty points across the material surface during the ablation test using an infrared thermometer.

Morphologies generated at the surface of the samples by the ablation treatment were evaluated from scanning electron microscopy images (FEI, Quanta 200 F, Brno, CZE).

\section{Results and Discussion}

\subsection{Ablation Tests}

Ablation tests of composite materials were conducted by placing the sample in the flame of an acetylene-oxygen torch. Both the change in linear dimensions and the mass loss were recorded. Results are shown in Table 1. Although values reported in the table are located above the acceptable limit $\left(0.14 \mathrm{~mm} \cdot \mathrm{s}^{-1}\right)$ [8], a large increase in the ablation resistance was observed when the phenolic resin was modified by the functionalized silica sols. This result can be used as a basis to increase the ablation resistance of formulations used in industrial practice. The ablation resistance of the composites decreased as follows: GPSD-PR > MPS-PR > GPS-MPS-PR > PR.

Table 1. Results of the ablation test for different composites.

\begin{tabular}{ccc}
\hline Matrix & Linear ablation $\left(\mathbf{m m} \cdot \mathbf{s}^{-\mathbf{1}}\right)$ & Mass ablation $\left(\mathbf{g} \cdot \mathbf{s}^{-\mathbf{1}}\right)$ \\
\hline GPSD-PR & 0.230 & 0.117 \\
MPS-PR & 0.268 & 0.125 \\
GPS-MPS-PR & 0.279 & 0.140 \\
PR & 0.316 & 0.405 \\
\hline
\end{tabular}

Composites based on GPSD-PR were the most stable to the flame with only two thirds of the linear ablation and only a quarter of the mass ablation observed for composites based on the neat PR. The enhanced performance is likely due to the covalent bonding of epoxy groups to the PR and to the fact that the thermal decomposition of epoxy matrices generates olefinic residues that can undergo carbonization [12]. Composites based on MPS-PR were next with regard to stability. This may be related to the reduced efficiency of the generation of covalent bonds with the PR through chain transfer reactions and to the propensity of the cross-linked methacrylate groups to thermally depolymerize at temperatures above $340{ }^{\circ} \mathrm{C}[24]$.

\subsection{Morphologies of Ablation Structures}

Examination of the ablation surfaces of the composites revealed that the generated pre-ceramic surface was glassy in appearance and that the material had liquified to some extent. It also showed that thermolysis generated pores in nearly every case, possibly through the evolution of gases while the 
material was in a molten state at the surface. The presence of porosity is a matter of concern because pores might allow flame or plasma to penetrate and damage the bulk of the composite.

Figure 2 shows two SEM images of the surfaces of different composites after the ablation test, at two different magnifications (70× in Figure $2 \mathrm{a}-\mathrm{d}$ and $1000 \times$ in Figure 2a'-d'). The smooth surface of the composite based on neat PR was converted to a porous and pitted surface after the ablation test (Figure 2a,a'), with glass fibers visible in some of the pores. The presence of aggregates inside the pores, coupled with porosity, could limit the use of this material as an ablation composite.

Composites made with the formulations containing silica sols led to denser structures of the ablation surfaces although pores were still present in every sample. The composite with the GPSD-PR matrix (Figure 2b, $b^{\prime}$ ) showed the most homogeneous surface, a fact that can be correlated with its better ablation behavior. Composites modified with methacrylate-functionalized silica sols (Figure $2 \mathrm{c}, \mathrm{c}^{\prime}, \mathrm{d}, \mathrm{d}^{\prime}$ ) showed the presence of partially collapsed bubbles at the surface of the specimens. These bubbles could be due to the rapid thermal depolymerization of methacrylate networks [24], a fact that can produce the exposure of the internal surface to the flame. This might be the reason for the lower ablation performance of the composites derived from methacrylate-functionalized silica sols.

Figure 2. SEM images of the ablation surfaces of different composites (a-d: $70 \times$ magnification; $\mathbf{a}^{\prime}-\mathbf{d}^{\prime}: 1000 \times$ magnification); matrices are neat phenolic resin (PR) (a and $\mathbf{a}^{\prime}$ ); GPSD-PR (b and $\mathbf{b}^{\prime}$ ); MPS-PR (c and $\mathbf{c}^{\prime}$ ); and GPS-MPS-PR (d and $\mathbf{d}^{\prime}$ ).
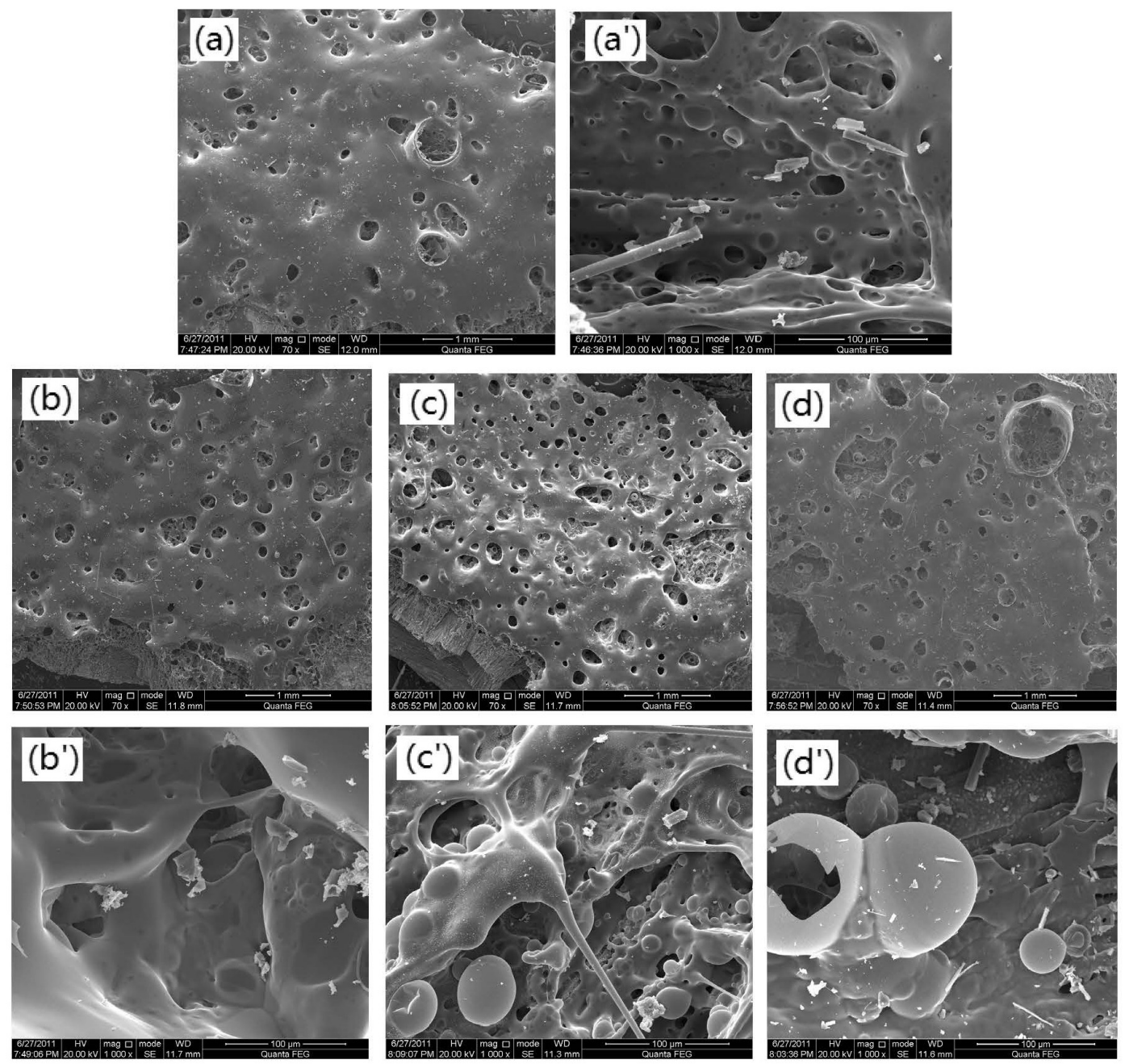


\subsection{Temperature Evolution during Ablation Tests}

Figure 3 shows the temperature evolution at the surface of different composites during the ablation tests. After $12 \mathrm{~s}$ of exposure to the flame, the composite made with the GPSD-PR matrix reached a surface temperature that was about $100{ }^{\circ} \mathrm{C}$ higher than the corresponding temperatures determined for the composites synthesized with the other two modified phenolic resins. This implied that the generated surface layer of degradation products acted as a better thermal insulator that retarded the heat flow into the sample. Again, this test evidenced the better performance of the phenolic matrix modified with the epoxy-functionalized silica sol.

Figure 3. Evolution of the ablation temperature at the surface during the ablation tests for composites obtained with the following matrices: GPSD-PR (a); MPS-PR (b); and GPS-MPS-PR (c).


\section{Conclusions}

A commercial phenolic resin was modified with three different functionalized silica sols and appropriate hardeners, in an attempt to increase the ablation resistance of their glass fiber composites. A significant improvement was observed when using $20 \mathrm{wt} \%$ of an epoxy-functionalized silica sol including also an epoxy monomer (DGEBA) and a hardener (EDA). A cross-linked thermoset was generated by reaction of epoxy groups with both the hardener and the phenolic hydroxyls of the resin. The mass ablation rate decreased from $0.405 \mathrm{~g} \cdot \mathrm{s}^{-1}$ for the neat phenolic resin to $0.117 \mathrm{~g} \cdot \mathrm{s}^{-1}$ for the modified matrix. The linear ablation rate decreased from 0.316 to $0.230 \mathrm{~mm} \cdot \mathrm{s}^{-1}$. Although this last value was higher than the acceptable limit required for commercial use, there are several parameters that can be optimized to improve the ablation resistance. For example, variables that can be manipulated are the type and proportion of hardener used in the formulation, the ratio among GPMS, TEOS and DGEBA, the proportion of the modifier with respect to the phenolic resin and the cure cycle.

Composites synthesized with methacrylate-functionalized silica sols showed a lower ablation resistance than those derived from the epoxy-functionalized sols. This was ascribed to the rapid depolymerization of networks based on methacrylate groups when exposed to the flame, a fact that generated partially collapsed bubbles at the surface and channels that gave easy access to the interior of the sample.

The improvement of the ablation resistance of the composite obtained with the epoxy-functionalized silica could be correlated with the higher temperatures generated at the surface during the ablation tests. After $12 \mathrm{~s}$ of exposure to the flame, the surface temperature of the specimen was about $100{ }^{\circ} \mathrm{C}$ higher than the corresponding temperatures determined for the two other formulations. This means that 
the surface layer made of degradation products acted as a better thermal insulator, protecting the material located at the bulk. Although SEM images revealed the presence of pores at the ablated surface of every specimen, the material modified with the epoxy-functionalized silica sol exhibited a lower porosity and a more homogeneous surface texture, correlating again with its better ablation resistance.

\section{Acknowledgments}

The financial supports from the Aerospace Supporting Fund (GN: 2012-HT-HGD-11); the Invitation of Foreign Experts Program of the Chinese Foreign Experts Bureau (GN: GDW20122300078); Natural Science Foundation of HLJ Province (Key Program, GN: ZD20080201); Excellent Youth Foundation of HLJ Province (GN: JC200902) and the 9th One-Thousand Expert Plan of The Organization Ministry of the Central Government (WQ20122300100), China, are gratefully acknowledged.

\section{Conflicts of Interest}

The authors declare no conflict of interest.

\section{References}

1. Rosensweig, R.E.; Beecher, N. Theory for the ablation of fiberglass-reinforced phenolic resin. AIAA J. 1963, 1, 1802-1809.

2. Geier, M. Space refractory materials. Metaux Corros. Idustrie 1969, 523, 92-128.

3. Koo, J.H.; Stretz, H.; Weispfenning, J.T.; Luo, Z.; Wootan, W. Nanocomposite Rocket Ablative Materials: Processing, Microstructure and Performance. In Proceedings of 45th AIAA/ASME/ ASCE/AHS/ASC Structures, Structural Dynamics \& Materials Conference, Palm Springs, CA, USA, 19-22 April 2004.

4. Koo, J.H.; Pilato, L.A.; Wissler, G.E. Polymer nanostructured materials for propulsion systems. J. Spacecr. Rockets 2007, 44, 1250-1262.

5. Li, W.; Liu, F.; Wei, L.; Zhao, T. Synthesis, morphology and properties of polydimethylsiloxanemodified allylated novolac/4,4'-bismaleimidodiphenylmethane. Eur. Polym. J. 2006, 42, 580-592.

6. Hung, A.Y.C.; Wang, F.Y.; Yeh, S.R.; Chen, W.J.M.; Ma, C.C. Carbon/carbon composites derived from poly(ethylene oxide)-modified novolac-type phenolic resin: Microstructure, physical and morphological properties. J. Appl. Polym. Sci. 2002, 84, 1609-1619.

7. Gloria, A.; Ronca, D.; Russo, T.; D’Amora, U.; Chierchia, M.; de Santis, R.; Nicolais, L.; Ambrosio, L. Technical features and criteria in designing fiber-reinforced composite materials: from the aerospace and aeronautical field to biomedical applications. J. Appl. Biomater. Biomech. 2011, 9, 151-163.

8. Winya, N.; Boonpan, A.; Prapunkarn, K. Study of factors affecting the ablation rate of phenolic resin/fiber glass. Int. J. Chem. Eng. Appl. 2013, 4, 234-237.

9. Qiu, J.; Cao, X.; Tian, C.; Zhang, J. Ablation performance of a novel super-hybrid composite. J. Mater. Sci. Technol. 2005, 21, 269-273.

10. Zhang, X.; Hu, L.; Sun, D. Nanoindentation and nanoscratch profiles of hybrid films based on ( $\gamma$-methacrylpropyl)trimethoxysilane and tetraethoxysilane. Acta Mater. 2006, 54, 5469-5475.

11. Baney, R.H.; Itoh, M.; Sakakibara, A.; Suzuki, T. Silsesquioxanes. Chem. Rev. 1995, 95, 1409-1430. 
12. Chiang, C.L.; Ma, C.C.M.; Wu, D.L.; Kuan, H.C. Preparation, characterization, and properties of novolac-type phenolic/ $\mathrm{SiO}_{2}$ hybrid organic-inorganic nanocomposite materials by sol-gel method. J. Appl. Polym. Sci. A 2003, 41, 905-913.

13. Zhang, X.; Hu, L.; Sun, D.; Zhao, W. Three-dimensional configurations of organic/inorganic hybrid nanostructureal blocks: A quantum chemical investigation for cage structure of ( $\gamma$-glycidyloxy)propylsilsesquioxane. J. Mol. Struct. 2008, 872, 197-204.

14. Chen, P.; Hu, L.; Zhang X.; Sun, D. Enhanced corrosion resistance for silsesquioxane coatings by diglycidyl ether of bisphenol A. Mater. Sci. Pol. 2007, 25, 843-849.

15. Wu, K.; Song, L.; Hu, Y.; Lu, H.; Kandola, B.K.; Kandare, E. Synthesis and characterization of a functional polyhedral oligomeric silsesquioxane and its flame retardancy in epoxy resin. Prog. Org. Coat. 2009, 65, 490-497.

16. Fasce, D.P.; Williams, R.J.J.; Erra-Balsells, R.; Ishikawa, Y.; Nonami. H. One-step synthesis of polyhedral silsesquioxanes bearing bulky substituents: UV-MALDI-TOF and ESI-TOF mass spectrometry characterization of reaction products. Macromolecules 2001, 34, 3534-3539.

17. Williams, R.J.J.; Erra-Balsells, R.; Ishikawa, Y.; Nonami, H.; Mauri, A.N.; Riccardi, C.C. UV-MALDI-TOF and ESI-TOF mass spectrometry characterization of silsesquioxanes obtained by the hydrolytic condensation of ( $\gamma$-glycidoxypropyl)trimethoxysilane in an epoxidized solvent. Macromol. Chem. Phys. 2001, 202, 2425-2433.

18. Eisenberg, P.; Erra-Balsells, R.; Ishikawa, Y.; Lucas, J.C.; Nonami, H.; Williams, R.J.J. Silsesquioxanes derived from the bulk polycondensation of [ $\gamma$-(methacryloxy)propyl] trimethoxysilane with concentrated formic acid: Evolution of molar mass distributions and fraction of intramolecular cycles. Macromolecules 2002, 35, 1160-1174.

19. Liu, Y.; Wang, D.; Hu, L.; Liu, J. Study on Phenolic-Resin/Carbon-fiber Ablation Composites Modified with Polyhedral Oligomeric Silsesquioxanes. In Proceedings of the 4th Annual IEEE International Conference on Nano/Micro Engineered and Molecular Systems, Shenzhen, China, 5-8 January 2009; pp. 605-608.

20. Pascault, J.P.; Sautereau, H.; Verdu, J.; Williams, R.J.J. Thermosetting Polymers; Marcel Dekker: New York, NY, USA, 2002; pp. 24-29.

21. Smith, M.E.; Ishida, H. Kinetics of the condensation reaction of epoxide with phenol: Linear chain growth versus branching. Macromolecules 1994, 27, 2701-2707.

22. Al Dwayyan, A.S.; Khan, M.N.; Salhi, S.A. Optical characterization of chemically etched nanoporous silicon embedded in sol-gel matrix. J. Nanomater. 2012, 2012, 1-7.

23. Loy, D.A.; Baugher, B.M.; Baugher, C.R.; Schneider, D.A.; Rahimian, K. Substituent effects on the sol-gel chemistry of organotrialkoxysilanes. Chem. Mater. 2000, 12, 3624-3632.

24. Joseph, P.; Tretsiakova-Mcnally, S. Reactive modifications of some chain-and step-growth polymers with phosphorus-containing compounds: Effects on flame retardance-a review. Polym. Adv. Technol. 2011, 22, 395-406.

(C) 2014 by the authors; licensee MDPI, Basel, Switzerland. This article is an open access article distributed under the terms and conditions of the Creative Commons Attribution license (http://creativecommons.org/licenses/by/3.0/). 